\title{
The narrowing olfactory landscape of insect odorant receptors
}

\author{
Jonathan D. Bohbot ${ }^{1 *}$ and Ronald J. Pitts ${ }^{2}$ \\ ${ }^{1}$ Department of Entomology, The Hebrew University, Rehovot, Israel, ${ }^{2}$ Department of Biological Sciences and Institute for \\ Global Health, Vanderbilt University, Nashville, TN, USA
}

The molecular basis of odorant detection and its corollary, the task of the odorant receptor, are fundamental to understanding olfactory coding and sensory ecology. Based on their molecular receptive range, olfactory receptors have been classified as pheromone and non-pheromone receptors, which are respectively activated by a single pheromone component ("specialist") or by multiple odorant ligands ("generalist"). This functional distinction is unique among ligand-gated ion channels and has shaped how we model olfactory coding both at the peripheral and central levels. Here, we revisit the long-standing combinatorial theory of olfaction and argue, based on physiological, pharmacological, evolutionary, and experimental grounds that the task of the odorant receptor is not different from that of neurotransmitter receptors localized in neuronal synapses.

Swedish University of Agricultural

Sciences, Sweden

Reviewed by:

Marcus Carl Stensmyr,

Lund University, Sweden Julien Pelletier,

Keele University, UK

Carolina Gomez-Diaz,

University of Konstanz, Germany

*Correspondence:

Jonathan D. Bohbot,

Department of Entomology, The

Robert H. Smith Faculty of Agriculture,

Food and Environment, The Hebrew

University, P.O. Box 12,

Rehovot 76100, Israel

jonathan.bohbot@mail.huji.ac.il

Specialty section:

This article was submitted to Chemical

Ecology, a section of the journal

Frontiers in Ecology and Evolution

Received: 29 January 2015

Accepted: 26 March 2015

Published: 10 April 2015

Citation:

Bohbot JD and Pitts RJ (2015) The narrowing olfactory landscape of

insect odorant receptors.

Front. Ecol. Evol. 3:39

doi: $10.3389 /$ fevo.2015.00039

Keywords: odorant receptor, ligand-gated ion channel, synaptic communication, olfactory communication, neurotransmitter receptors

\section{Introduction}

How insects process odorants is a central question in the field of olfactory neurobiology. The number of green leaf volatiles (GLVs) produced by plants and volatile organic compounds (VOCs) emitted by animals or other sources (rotting fruits and excretion products) are not well-defined. Most recent studies report that GLVs (Dudareva et al., 2006; Knudsen et al., 2006) and VOCs number just below 2000 chemicals (Penn et al., 2007; Phillips et al., 2013; de Lacy Costello et al., 2014) (Figure 1A), which is likely an underestimate. Insects rely on odorant receptor (Or), ionotropic receptor (Ir), and the $\mathrm{CO}_{2}$-sensing gustatory receptor $(\mathrm{Gr})$ gene families for the long-range detection of airborne chemical cues (Suh et al., 2014). Until now, the Or clade has been the most extensively studied from both evolutionary and functional standpoints, and will be the focus of this theory article.

Odorant Receptors (ORs) are at the front line of odorant detection and much like neurotransmitter receptors (NRs), their task is to convert chemical signals into electrical outputs (Figure 1B) thereby ensuring the continuity of information flowing from the environment to the brain. Functional ORs are heteromeric complexes composed of an odorant-sensing unit belonging to a large and diverse family and a conserved OR co-receptor named ORco (for review, see Suh et al., 2014). These OR complexes are localized in the dendrites of olfactory receptor neurons (ORNs) embedded in sensilla, which project from the insect cuticular surface on olfactory appendages.

Insect OR repertoires vary greatly in number, ranging from 0 in the bristletail (Missbach et al., 2014), 110 in the yellow fever mosquito Aedes aegypti (Bohbot et al., 2007), 170 in the honeybee Apis mellifera (Robertson and Wanner, 2006) to over 400 ORs in eusocial ants (Zhou et al., 2012). Despite such a limited number of ORs, insects navigate complex chemical environments by 


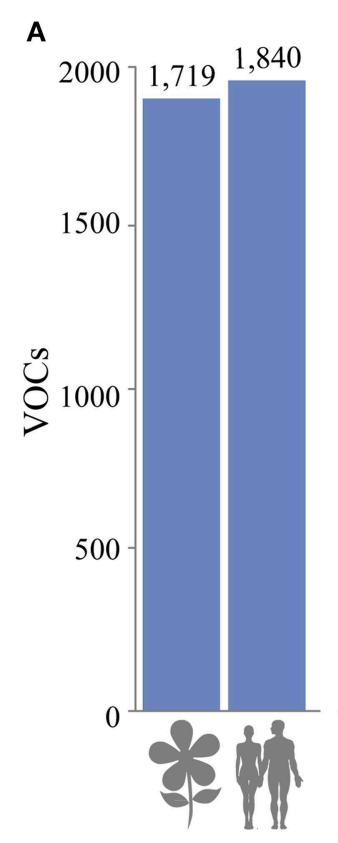

Flower Host
B
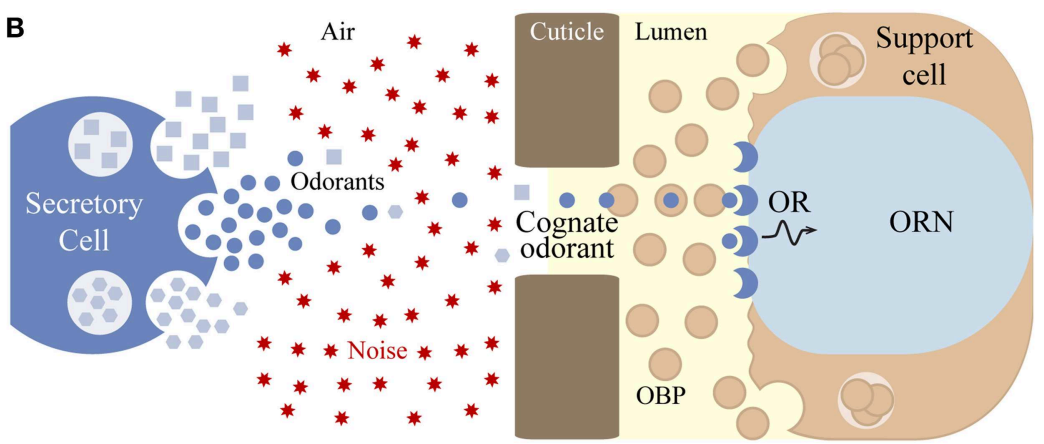

$\stackrel{\text { Odor source }}{\longleftrightarrow}$

Space

Receiver

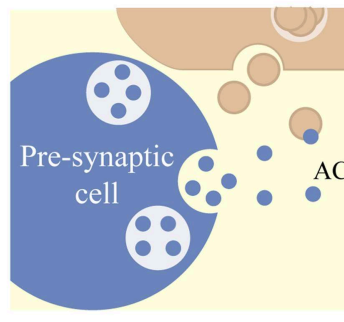

\section{FIGURE 1 | Ecological context of olfactory and synaptic}

communications. (A) Human and plant volatile organic compounds (VOCs) number in the thousands, of which an unknown number of odorants are involved in animal-insect and plant-insect interactions. By contrast, few neurotransmitters and co-transmitters are present in the synaptic cleft.

(B) Synaptic and olfactory communications are tripartite systems: large quantities of chemicals are secreted by an odor source (e.g., a secretory cell or organic matter), come into contact with binding proteins secreted by glia or support cells and activate receptors on the surface of receiver cells, which convert these chemical signals into electrical outputs. The main difference between olfactory and synaptic communications is that the concentration of cognate ligands that reach neurotransmitter receptors (NRs) and odorant receptors (ORs) is hypothesized to be orders of magnitude different. In cholinergic synapses, glia release acetylcholine-binding protein (AChBP) to capture acetylcholine (Ach) and suppress synaptic transmission. The hydrophobic nature of odorants may suffice to keep the sensillum lymph relatively free of chemical noise while odorant-binding proteins (OBPs) capture ecologically relevant odorant to foster OR activation. exhibiting remarkable olfactory sensitivity, considering that some of these airborne VOCs are present in the air in the picomolar range (Phillips, 1997). The impact of this information on insect fitness, both in terms of survival and reproduction cannot be overstated. Host species must be located, conspecifics recognized, trails followed, and potential dangers avoided during many stages of the insect life cycle. Thus, it is tempting to surmise that ORs have evolved high sensitivity and selectivity capabilities for the detection of ecologically meaningful odorants.

The combinatorial theory of olfaction (Malnic et al., 1999) explains how this limited set of "generalist" ORs, thereafter referred to as non-pheromone receptors (nPRs), encode thousands of odorants. This weak shape theory (Rinaldi, 2007), a "relaxed" version of the stereochemical model of olfaction (Moncrieff, 1949; Amoore, 1963), postulates that an nPR only recognizes a part of the odorant and therefore can accommodate a variety of odorants provided they share common chemical features. Functional studies on Drosophila melanogaster (Hallem et al., 2004) and Anopheles gambiae (Carey et al., 2010; Wang et al., 2010) support the theory that nPRs exhibit broad molecular receptivity. However, moth pheromone receptors (PRs) function according to a more rigid lock-and-key mechanism as they are activated by single pheromone components (Große-Wilde et al.,
2007; Miura et al., 2009; Wang et al., 2011; Leary et al., 2012; Sun et al., 2013). Together, nPRs and PRs allow the peripheral olfactory system to encode the identity and quantity of odorants over a wide range of molecules and concentrations. This model continues to have important implications on the conceptual roles of the peripheral and central nervous systems in terms of signal filtering and olfactory coding, respectively.

In recent years, the discovery of an increasing number of specialized nPRs in moths and mosquitoes has broken the pharmacological monopoly of PRs in terms of sensitivity and specificity. This recent development is cause for revisiting the concepts of molecular receptive range and olfactory tuning as well as their respective contributions to the theory of combinatorial receptor codes for odorants in insects. This article continues an earlier discussion on the task of the OR (Bohbot and Dickens, 2012a). Here, we have compared the physiological contexts and pharmacological properties of ORs and NRs by discussing the notions of receptive range and olfactory tuning from the perspective of chemical ecology and evolution and review the experimental designs supporting the notion of generalist ORs, which ultimately have shaped the combinatorial theory of olfaction. Finally, we comment on the respective roles of the peripheral and central nervous systems in olfactory coding and propose strategies for testing our ideas. 


\section{Olfactory and Synaptic Communications}

The foundation of our discussion begins with a deceptively simple question: is the task of an insect OR different from that of a NR (Firestein, 2001)? Although not the focus of this article, ionotropic glutamate receptors provide a clear example of an evolutionarily ancient mechanism linking olfactory and synaptic communications (Croset et al., 2010). In both cases, a chemical signal travels through space to interact with binding-proteins and degrading enzymes (Vogt and Riddiford, 1981; Smit et al., 2001), which modulate the signal before being detected by an ionotropic receptor (Figure 1B). Despite these similarities, the chemical constraints on ORs and NRs differ in one fundamental way. In the case of synaptic neurotransmission, millimolar concentrations of water-soluble neurotransmitters are released in the synaptic cleft (Kuffler and Yoshikami, 1975; Clements, 1996; Karayannis et al., 2010), a space several nanometers wide (Stocker and Nuesch, 1975; Felten and Olschowka, 1987) whose chemical content is regulated by the local cellular environment. Synaptic clefts may contain one or more neurotransmitters and co-transmitters (Figure 1B), but their exact compositions remain largely unknown (Burnstock, 2004). Considering that these conditions are optimal for synaptic transmission, it may be sufficient that NRs act as low-sensitivity receptors (millimolar range) much like the Bombyx mori GR9, a highly selective sugar receptor activated by millimolar concentrations of $\mathrm{D}$-fructose that functions as an ionotropic receptor (Sato et al., 2011) (Figure 2A). Moreover, low-sensitivity in the synapse reduces potential noise associated with spontaneous neurotransmitter release (Faisal et al., 2008).

By comparison, ORs potentially face a far more complex chemical environment. With greater distances form the emitting sources and due to the chaotic nature of turbulent air, the occurrence of any particular cognate odorant reaching an $\mathrm{OR}$ is orders of magnitude lower than the probability of neurotransmitters interacting with NRs. The presence of odorant molecules competing for the recognition sites of ORs is a matter of conjecture but it is likely that due to their hydrophobic nature, most odorants do not cross the sensillum lymph barrier unless helped by transporter proteins such as odorant-binding proteins (Figure 1B). Provided that these odorant-binding proteins are selective, the sensillum lymph and synaptic cleft may therefore be similar in terms of chemical complexity. To increase selective OR-odorant binding probability events, insects have evolved several anatomical and biochemical adaptations including elongated porous sensillae and binding proteins to facilitate the transport of cognate odorants through the sensillum lymph. Based on these parameters, we suggest that ORs are likely to be more sensitive and equally specific toward their cognate ligands than NRs.

\section{Comparative Pharmacology of ORs and NRs}

How do ligand-receptor interactions differ in terms of sensitivity and specificity in the context of olfactory and synaptic communications? To address this question, we have compared these pharmacological features between ORs and other ligand-gated ion channels (LGICs) including cys-loop receptors, ionotropic glutamate receptors, and ATP-gated channels. We surveyed the scientific literature and compared 50 data points representing the $\mathrm{EC}_{50}$-values (concentration of ligand that elicits $50 \%$ of maximum receptor activation) of NRs, nPRs, PRs, and one sugar GR (Supplementary Table 1) expressed in Xenopus laevis oocytes (Figure 2A). This expression system provided the largest $\mathrm{EC}_{50}$ dataset of LGICs.

NRs exhibit sensitivities in the nanomolar and micromolar range. Nicotinic acetylcholine receptors (nAChRs) have the lowest and broadest range of sensitivities to acetylcholine, which is likely caused by the use of non-native subunit combinations (Chavez-Noriega et al., 1997; Wonnacott and Barik, 2007). Ligands for the serotonin, glutamate, GABA, and N-methyl-Daspartate receptors display relatively narrower potencies in the nanomolar range (Figure 2A), which, contrary to our assumption, shows that LGICs are highly sensitive to their cognate ligand.

The majority of PRs also exhibit $\mathrm{EC}_{50}$-values in the nanomolar range with the moths Ostrinia furnicalis OR3 showing the highest sensitivity to (Z)-11-tetradecenyl acetate (Leary et al., 2012) and Heliothis armigera OR13 showing the lowest sensitivity to (Z)11-hexadecenal (Liu et al., 2013) (Figure 2A). All the nPRs for which cognate ligands have been identified using sensory physiology and behavior exhibit $\mathrm{EC}_{50}$-values in the nanomolar range as well, suggesting that the distinction between PRs and nonPRs on the bases of sensitivity is unwarranted provided that the cognate ligands are used. Recently, a "generalist" OR has been shown to detect the GLV E- $\beta$-farnesene in the nanomolar range demonstrating at the molecular level that GLV-sensing ORs can be highly sensitive and specific (Liu et al., 2014).

Receptor specificity is the ability to distinguish between a cognate odorant ligand and its closest structural analog. We used $\mathrm{EC}_{50}$-values to benchmark the discriminative power of PRs and nPRs, an aspect of LGIC pharmacology that has been scarcely studied, which limits our ability to draw general conclusions. Nonetheless, we find examples of ORs that display clear differential sensitivities ranging from 1 to 2 orders of magnitude (Figure 2B), suggesting that these receptors have evolved high stereospecificity, which likely reflect their ecological relevance (Bohbot and Dickens, 2009). Several mosquito ORs show superior recognition abilities (Hughes et al., 2010; Pelletier et al., 2010) compared to some moth PRs, which discriminate between geometric pheromone isomers (Wanner et al., 2010; Leary et al., 2012). Considering how little to no recognition PRs and nPRs display toward structural analogs of the cognate odorant, it is interesting to note that some PRs are more robustly activated by the formate analogs of known aldehyde constituents of moth pheromones (Xu et al., 2012). This observation might suggest that "super" ligands may be discovered by exploring close structural analogs of known cognate ligands. However, the selectivity of the octenol receptor (OR8) from A. aegypti suggests that any modifications to the cognate ligands elicit little to no receptor activation (Bohbot and Dickens, 2009). Whatever the case may be, detailed studies of formate pheromone derivatives will help clarify our understanding of receptor-ligand molecular relationships and may lead to the development of novel 


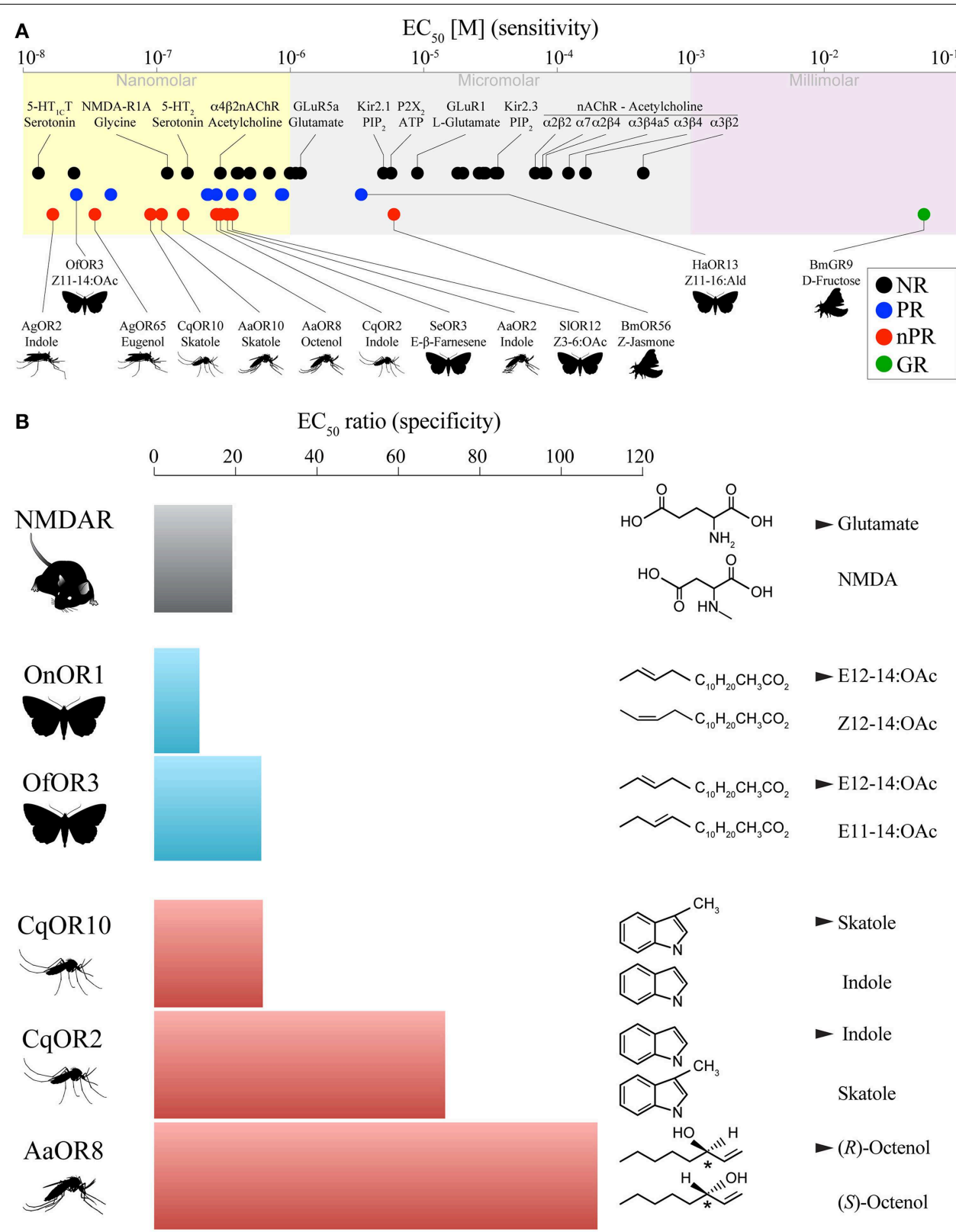

FIGURE 2 | Comparative pharmacology of ligand-gated ion channels and odorant receptors. (A) The sensitivity of a receptor toward its natural ligand is characterized by the Effective Concentration $50\left(E_{50}\right)$, which is the concentration eliciting $50 \%$ of the receptor maximal response. Except for many acetylcholine receptors and the gustatory receptor 9 (GR9), the sensitivities of neurotransmitter receptors (NRs), pheromone receptors (PRs) and non-pheromone receptors (nPRs) are in the nanomolar range. (B)
Receptor specificity ( $\mathrm{EC}_{50}$ ratio) is a measure of receptor preference between a cognate ligand and a related structural analog. For instance, NMDAR requires 19 times more NMDA to reach the activation level elicited by glutamate. Some nPRs exhibit higher specificity than some PRs and the $\mathrm{N}$-methyl-D-aspartate receptor (NMDAR). Cognate ligands are indicated by a solid triangle. References and species name abbreviations can be found in Supplementary Table 1. bioactive molecules affecting the behavior of agricultural and medical pests.

\section{ORs as Ecological Adaptations}

The combinatorial receptor coding scheme posits that the majority of ORs, with the exclusion of PRs, are promiscuous in terms of odorant recognition. While it provides an attractive model to encode a wide variety of odorant cues, it is counterintuitive from an evolutionary standpoint. Such an olfactory system would potentially be exposed to continuous overstimulation and thus lack the ability to distinguish important signals from background noise. In addition, the central nervous system would be required to filter this information into a useful code. What evolutionary 
mechanism would explain how olfactory systems develop such a state?

Distinctive features of insect ORs include high evolutionary rates (Neafsey et al., 2015), lineage-specific expansions (Hill et al., 2002; Robertson and Wanner, 2006; Zhou et al., 2012; Cande et al., 2013) and large variations in gene repertoires (Nei et al., 2008; Sánchez-Gracia et al., 2009). The major mechanism of evolution of the $\mathrm{Or}$ gene family follows the birth-and-death model (Sánchez-Gracia et al., 2009) whereby genes multiply via tandem gene duplication events and are removed by deletion (Gardiner et al., 2008). Studies on Or evolution in Drosophila (McBride, 2007; McBride et al., 2007; Gardiner et al., 2008; Sánchez-Gracia et al., 2009; Stensmyr et al., 2012) and Anopheles mosquitoes (Neafsey et al., 2015) indicate that the birth-and-death of $\mathrm{Or}$ genes is not random (McBride et al., 2007) but that genetic diversity and variability are principally acted upon by purifying selection. Despite these observations, Or genes exhibit some of the highest level of positive selection in many lineage specific expansions suggesting functional divergence associated with host specialization (McBride et al., 2007; Neafsey et al., 2015), and mate selection (Leary et al., 2012).

The mosquito-specific indole receptors show remarkable sequence conservation across the Culicinae and Anophelinae families (Bohbot et al., 2007), indicating that they fulfill fundamental olfactory functions critical to the life cycle of these insects. These receptors diversified through several instances of duplication events followed by positive selection, which diversified their tuning range toward indole analogs, functions that have subsequently been maintained by purifying selection. It appears that although the evolutionary mechanisms differ, lineage-specific ORs are associated with ecological adaptations (Figure 3). The evolution of the mosquito A. aegypti OR4 illustrates how these evolutionary forces also act on a short time scale: alleles of OR4 in A. aegypti sub-species exhibit different sensitivities toward the cognate human odorant sulcatone, which is associated with human host preference (McBride et al., 2014). Mate selection is also driving odorant tuning in closely related noctuid species, where positive selection is acting on discrete amino-acid residues of PRs (Leary et al., 2012).

The constant tuning of ORs occurring over short and long time scales, as well as the conservation of specific lineages via purifying selection, runs counter to the notion that these receptors possess promiscuous binding sites. Such a broad peripheral filter would provide the central nervous system with the greater task of sorting out this crude input. However, there is little evidence that the CNS and the antennal lobe accomplish this task (Sachse and Galizia, 2003). In fact, one study has demonstrated that the discriminatory capabilities of the antennal lobes diminish when stimulated by high concentrations of odorants (Silbering et al., 2008). Rather, we concur with previous authors that ORs are better chemical detectors than previously assumed and that the burden of extracting behaviorally relevant odorant signals from the environment is largely their task (Hansson and Stensmyr, 2011). If so, the role of the antennal lobes would be to integrate olfactory inputs from hardwired lines associated with the detection of cognate odorants.

\section{Molecular Receptive Range and Olfactory Tuning}

The inherent advantages of the two-electrode voltage clamp system (Kvist et al., 2011) has facilitated the use of oocytes for assaying OR-odorant interactions (see References in Supplementary Table 1). Despite these efforts, the oocyte system is limited in the number of chemicals that can be tested and is most efficiently

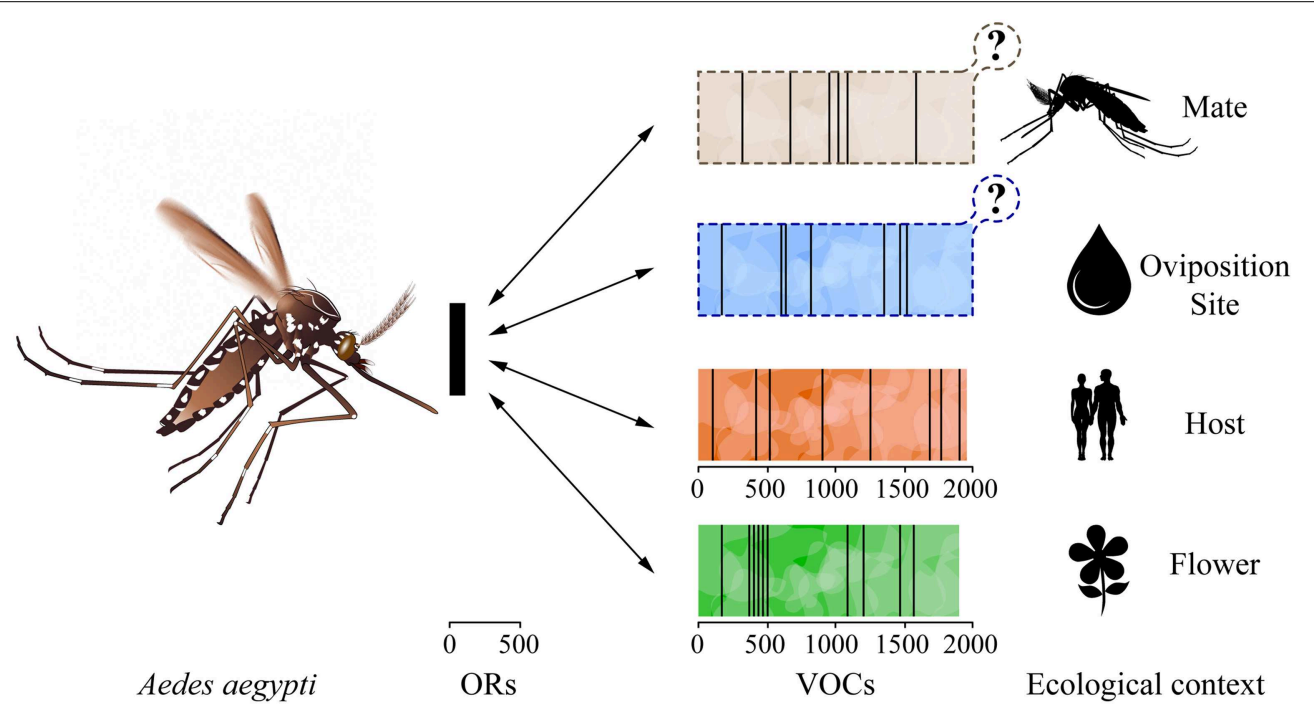

FIGURE 3 | Odorant receptors are molecular adaptations to various ecological niches. The mosquito Aedes aegypti may use up to 110 odorant receptors (ORs) to detect specific behaviorally relevant odorants (vertical black bars) emitted in the context of various ecological contexts. For

illustration purposes, these cognate odorants are unknown and have been placed arbitrarily on the scale. The number of volatile organic compounds (VOCs) emitted by potential mates and oviposition sites remains largely unknown. 
used for target validation studies or studies of receptor-ligand qualities such as concentration responsiveness and comparative efficacies (Kvist et al., 2011). Where oocytes have been used as a screen for previously uncharacterized insect ORs, dozens of receptors and tens of odorants have been used, providing hundreds of receptor-ligand comparisons (Wang et al., 2010). In stark contrast are functional studies in Human Embryonic Kidney cells, which can be used to screen tens of thousands of ligandreceptor combinations (Rinker et al., 2012). Both systems suffer from the inherent requirement to solubilize odorants in aqueous solutions, making it difficult to include VOCs that are often highly insoluble. This limitation can be circumvented by using an in vivo system such as the Drosophila empty neuron where VOCs can be delivered via airstream to OSNs expressing heterospecific ORs (Dobritsa et al., 2003), but again this system lacks truly high throughput capacity.

Two of the most critical limitations of OR heterologous functional screens relate to the lack of chemical ecological context. The first limitation is the unavoidable gap in a priori information regarding potentially meaningful relationships between ORs and their cognate ligands. This usually means that OR functional studies are carried out in more or less random fashion with ORs being targeted by VOCs that happen to be readily available.

The second limitation concerns the use of very high concentrations of odorants that may produce "hits;" i.e., VOCs that activate ORs with low probability of having any adaptive value (Dobritsa et al., 2003; Carey et al., 2010; Wang et al., 2010; Ray et al., 2014). This conundrum may not be of concern if the goal of the study is simply to identify chemicals that activate ORs and can be used at high concentrations for studies of channel properties or perhaps to identify drugs that alter insect behavior. Indeed the OR screens that have been carried out to date have been extremely valuable in elucidating the mechanisms of OR function and in providing a broader framework from which we can continue to refine our understanding of insect olfaction (reviewed in Suh et al., 2014). However, if a hypothesis-driven study depends on understanding ORs in the context of chemical ecology, random screening is unlikely to produce meaningful information. There are excellent examples of insect ORs that can be activated by numerous VOCs at low millimolar/high micromolar concentrations, which would tend to support the conclusion those receptors are broadly tuned (Hallem and Carlson, 2004, 2006; Kreher et al., 2008; Carey et al., 2010; Wang et al., 2010). However, some receptors in the same screens appear to be much more narrowly tuned when the VOC concentrations are reduced to low micromolar/high nanomolar range. This would be expected for a natural receptor-ligand pairing. For example, AgORs 2 and 10 exhibit broad molecular receptivity at high VOC concentrations but are in fact highly sensitive and narrowly tuned to their apparent cognate ligands, indole and skatole, respectively (Wang et al., 2010). Moreover, follow up studies with these receptors have validated the conclusions that these receptors display highly selective responses with low $\mathrm{EC}_{50}$-values (Bohbot et al., 2011). These examples are analogous to a mammalian LGIC, the NMDA receptor, where glutamate is the endogenous ligand with high potency $\left(\mathrm{EC}_{50}=640 \mathrm{nM}\right)$, while a synthetic competitive agonist for which the receptor is named after exhibits a potency that is over an order of magnitude less potent $\left(\mathrm{EC}_{50}=12,000 \mathrm{nM}\right)$ (Nakanishi et al., 1992) (Figure 2B). In addition, the full activation of NMDA receptors in vivo requires the binding of glycine to an allosteric site and has a potency of $77 \mathrm{nM}$. These examples illustrate two mechanisms by which LGICs may display apparent broad molecular receptivity range: (i) high concentrations of non-meaningful orthosteric ligands and (ii) contributions of topographically distinct (allosteric) sites (Figure 4A). Interestingly, most studies of insect PRs in heterologous systems have not suffered from these kinds of biases, probably because their likely cognate ligands were previously known in pheromone blends (Nakagawa et al., 2005; Wanner et al., 2007, 2010; Mitsuno et al., 2008; Wang et al., 2011; Leary et al., 2012; Liu et al., 2013; Sun et al., 2013). One test of our theory on this topic would be to "screen" PRs with high concentrations of VOC libraries like the ones described in previous studies. We speculate that numerous chemicals would activate PRs in that situation, leading to the false perception that PRs are "generalists." Taken together, the limitations described here prescribe caution when interpreting the results of heterologous expression data and OR function. The dogma that many ORs are functional "generalists" has often been based on limited VOC libraries administered at high concentrations in heterologous systems. This problem is also encountered when screening ORNs in vivo using high doses (up to $10^{-2}$ dilutions) of odorants (Hallem et al., 2004; Hallem and Carlson, 2006; Carey et al., 2010). An important question to ask is whether one would expect to obtain such a high percentage of positive OR activating odorants among such small numbers of VOCs? In our opinion this seems unlikely and would lead us to conclude that such receptors in natural settings would be prone to activation by a potentially huge number of odorants that would render them quite useless at encoding meaningful information.

\section{Conclusions}

The physiological, pharmacological, evolutionary, and experimental arguments presented in this communication are part speculative and part empirical. Altogether, they support an idea that both ORs and NRs are specialized in the detection of evolutionary meaningful chemical cues and their distinction may remain based on their ecological context rather than on their pharmacological properties. To demonstrate this, we have provided evidence that (i) narrow tuning is not the exclusive attribute of PRs, (ii) GLVs and other non-pheromonal VOCs specifically activate nPRs, (iii) ORs are subjected to powerful selective pressures, and (iv) high concentrations of odorant stimuli cause broad non-specific OR responses.

Are ORs distributed on a continuum of tuning breadths made of a small number of narrowly tuned PRs and a majority of general nPRs (Figure 4B)? According to our analysis, ORs exhibit both broad molecular receptivity and narrow olfactory tuning. Insect ORs may be narrowly tuned to behaviorally relevant odorants but this selectivity can be overcome by overloading the system with high concentrations of chemicals carrying no adaptive value. Therefore, we distinguish the notions of molecular receptive range and olfactory tuning based on their chemical and ecological merits. 


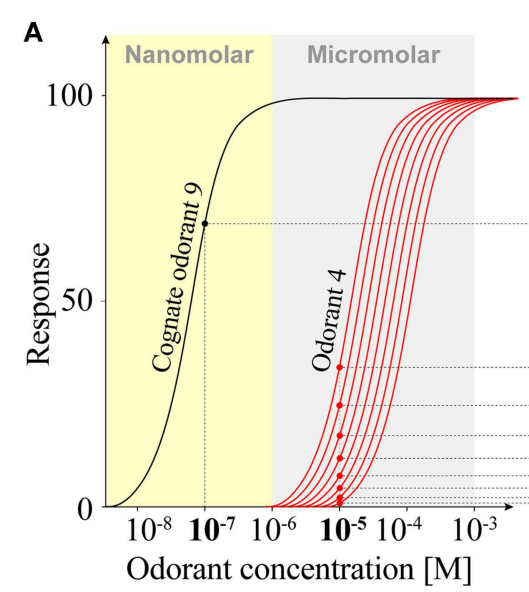

B

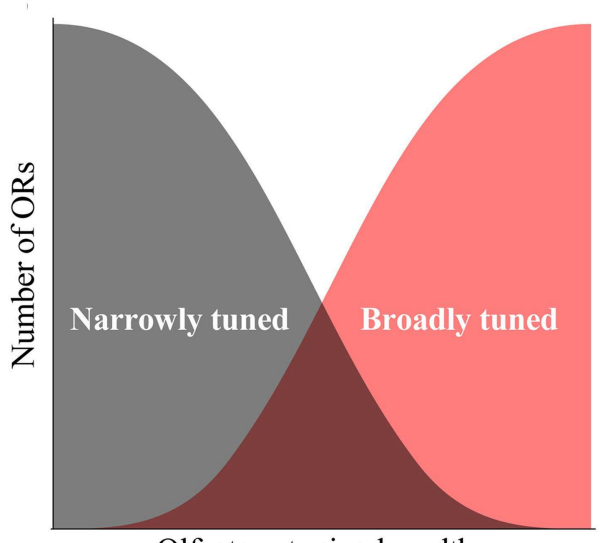

Olfactory tuning breadth
Olfactory tuning $\left[10^{-7} \mathrm{M}\right]$

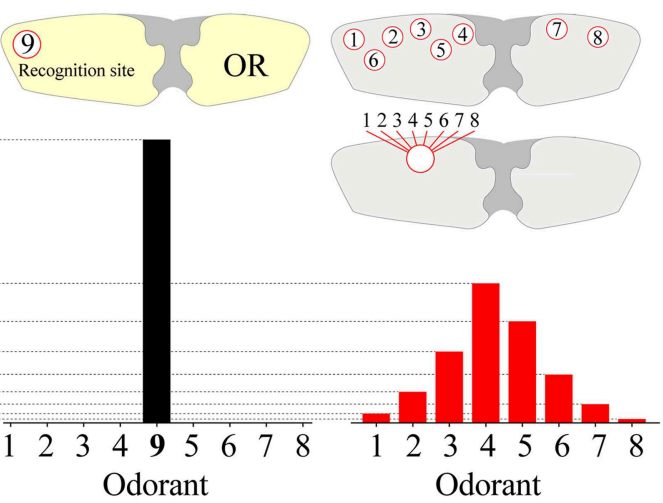

FIGURE 4 | Molecular receptive range and olfactory tuning of odorant receptors. (A) OR tuning curves represent OR responses to a set of odorants at a given concentration. Compared to odorant-response curves, tuning curves provide less resolution and sometime misleading information in terms of sensitivity and response profile. At high concentration $\left(10^{-5} \mathrm{M}\right)$, an odorant receptor (OR) is activated by odorants 1 through 8 thereby exhibiting a broad molecular receptive range (red histogram). These odorants may interact with an orthosteric site or in combination with multiple allosteric sites. At low concentration $\left(10^{-7} \mathrm{M}\right)$, the same OR is selectively activated by the cognate odorant 9 (black histogram) thus exhibiting a narrow olfactory tuning
C
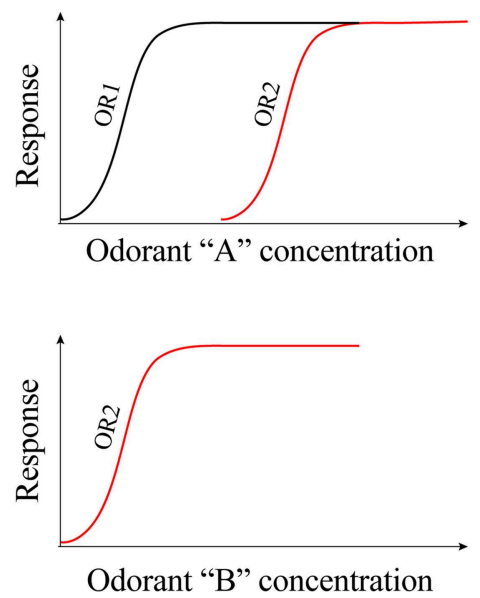

While this hypothesis does not exclude the possibility that a few ORs exhibit broad olfactory tuning, it provides greater emphasis to the possibility that most ORs may be narrowly tuned (Figure 4B). Furthermore, we propose that nanomolar EC 50- $^{-}$ values provide a criterion for insect OR deorphanization. If this is correct, the cognate ligands for Bombyx mori OR56 (Tanaka et al., 2009) and Helicoverpa armigera OR13 (Liu et al., 2013) remain to be identified (Figure 2A).

How do insects encode odorant intensity? It has been suggested that one of the mechanisms by which Drosophila ORs encode odorant intensity is by using paralogous OR pairs such as $42 \mathrm{a}$ and $42 \mathrm{~b}$, each detecting low and high amounts of the same odorant ligand (Kreher et al., 2008) (Figure 4C). This concept does not exclude the possibility that the response of an OR to high ligand concentrations rather indicates that the cognate ligand has yet to be identified. Such is the case with the mosquito indolergic receptors, OR2 and OR10, which respond to indole with high and low sensitivities, respectively (Bohbot et al., 2011). However, OR10 is narrowly tuned to skatole, a methylated analog of indole (Hughes et al., 2010; Bohbot and Dickens, 2012b). Perhaps more intriguing is the possibility that these two phenomena coexist. This is now a testable hypothesis using the aforementioned paralogous Drosophila ORs and looking for the cognate ligand of the low sensitivity receptor.

One of the greatest challenges in receptor neurobiology is the identification of OR-cognate odorant pairs. Based on our 


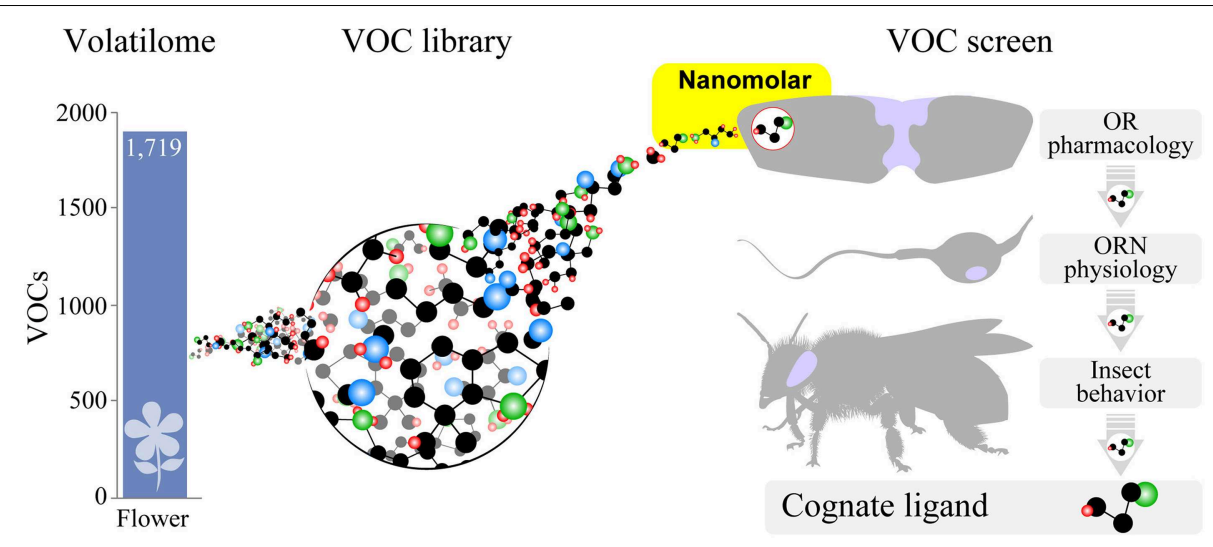

FIGURE 5 | Large-scale identification of behaviorally relevant volatile organic compounds. Animal and plant volatilomes may be used to generate a volatile organic compound (VOC) library for high throughput screening of odorant receptors (ORs) expressed in heterologous cell expression systems. VOCs exhibiting activity in the nanomolar range are further validated at the neurophysiological and behavioral levels. analysis, we conclude that ORs and NRs are specialized receptors that selectively detect low concentrations of cognate ligands in the context of chemical communication. However, the data presented here is only a snapshot of the insect OR family. Among a few species, only a handful of ORs have been deorphanized, i.e., for which a cognate ligand has been identified. Matching host or plant volatilomes to an OR repertoire will not be trivial but would offer a comprehensive strategy to identify additional OR-cognate odorant pairs. Many of these VOCs have been published (Knudsen et al., 2006; de Lacy Costello et al., 2014) and may be used to generate a VOC library to test the response of heterologously expressed ORs (Figure 5) (Bohbot et al., 2014). A similar strategy, using gas chromatography-coupled single sensillum recording, has been applied to discover that ORNs are narrowly tuned to GLVs (Binyameen et al., 2014; Suer, 2014). Both approaches may provide lead bioactive compounds for behavioral validation.

The notions of generalist and specialist receptors were first proposed in 1964 by Schneider et al. (Schneider, 1964) in the context of ORN activation and were later applied to ORs as their underlying molecular mechanisms. Earlier authors had reported that GLV-detecting ORNs were as specialized as sex pheromone receptor neurons (Kafka, 1987; Dickens, 1990; Anderson and Hansson, 1995). Anderson recognized that the use of a limited number of GLVs for physiological screens prevented the identification of behaviorally relevant odorant ligands. Likewise, we have underlined that current OR deorphanization efforts use between 80 and 100 odorants, which is at least three-fold lower than the number of VOCs insects encounter in nature. The pharmacological data currently available reveal that PRs and nPRs exhibit comparable functional specialization provided the correct cognate ligands are known (Hansson and Stensmyr, 2011; Bengtsson et al., 2014).

The combination of short lifespan and narrow behavioral complexity in insects may explain the evolution of a peripheral olfactory system tuned to a narrow range of odorants involved with reproduction, mate selection and food selection, which do not exclude the possibility of associative learning. Interestingly, mammals can learn to detect chemicals devoid of adaptive value with high sensitivity and specificity. However, the comparatively oversized olfactory epithelium of mammals combined with their sniffing behavior may compensate for the inherent lack of sensitivity of its OR repertoire. Indeed, detector dogs seem to only detect the most abundant volatile chemicals in the headspace of explosives (Harper et al., 2005). On the other hand, mammals may have developed a peripheral olfactory system with higher tolerance for a greater variety of odorants amenable to odorant-based associations (Leon and Johnson, 2003; Wilson and Stevenson, 2003). It is more likely that the complexity of the central nervous system account for most of the plasticity required for associative learning (Mandairon and Linster, 2008).

There is no expedient method to test the theory proposed herein. Indeed, the identification of cognate odorants is time consuming and resource intensive. Since the pioneering studies on the deorphanization of insect ORs (Hallem et al., 2004; Carey et al., 2010; Wang et al., 2010), targeted functional studies have identified cognate ligands for nPRs and it is reasonable to expect that more will be identified. What remains to be understood is indeed staggering and will have important consequences in the fields of olfactory coding, medicine, and agriculture.

\section{Acknowledgments}

The authors are grateful to Dr. Richard G. Vogt for his critical review of this manuscript.

\section{Supplementary Material}

The Supplementary Material for this article can be found online at: http://www.frontiersin.org/journal/10.3389/fevo.2015. 00039/abstract 


\section{References}

Amoore, J. E. (1963). Stereochemical theory of olfaction. Nature 198, 271-272. doi: $10.1038 / 198271 \mathrm{a} 0$

Anderson, P., and Hansson, B. S. (1995). Plant-odour-specific receptor neurones on the antennae of female and male Spodoptera littoralis. Physiol. Entomol. 20, 189-198. doi: 10.1111/j.1365-3032.1995.tb00001.x

Bengtsson, J. M., Gonzalez, F., Cattaneo, A. M., Montagné, N., Walker, W. B., Bengtsson, M., et al. (2014). A predicted sex pheromone receptor of codling moth Cydia pomonella detects the plant volatile pear ester. Front. Ecol. Evol. 2:33. doi: $10.3389 /$ fevo.2014.00033

Binyameen, M., Anderson, P., Ignell, R., Birgersson, G., Razaq, M., Shad, S. A., et al. (2014). Identification of plant semiochemicals and characterization of new olfactory sensory neuron types in a polyphagous pest moth, Spodoptera littoralis. Chem. Senses. 39, 719-733. doi: 10.1093/chemse/bju046

Bohbot, J. D., and Dickens, J. C. (2009). Characterization of an enantioselective odorant receptor in the yellow fever mosquito Aedes aegypti. PLoS ONE 4:e7032. doi: 10.1371/journal.pone.0007032

Bohbot, J. D., and Dickens, J. C. (2012a). Selectivity of odorant receptors in insects. Front Cell Neurosci. 6:29. doi: 10.3389/fncel.2012.00029

Bohbot, J. D., and Dickens, J. C. (2012b). Odorant receptor modulation: ternary paradigm for mode of action of insect repellents. Neuropharmacology 62, 2086-2095. doi: 10.1016/j.neuropharm.2012.01.004.

Bohbot, J. D., Jones, P. L., Wang, G., Pitts, R. J., Pask, G. M., and Zwiebel, L. J. (2011). Conservation of indole responsive odorant receptors in mosquitoes reveals an ancient olfactory trait. Chem. Senses 36, 149-160. doi: 10.1093/chemse/bjq105

Bohbot, J. D., Strickman, D., and Zwiebel, L. J. (2014). The future of insect repellent discovery and development. Outlook Pest Man. 25, 265-270. doi: 10.1564/v25_aug_05

Bohbot, J., Pitts, R. J., Kwon, H. W., Rutzler, M., Robertson, H. M., and Zwiebel, L. J. (2007). Molecular characterization of the Aedes aegypti odorant receptor gene family. Insect Mol. Biol. 16, 525-537. doi: 10.1111/j.1365-2583.2007.00748.x

Burnstock, G. (2004). Cotransmission. Curr. Opin. Pharmacol. 4, 47-52. doi: 10.1016/j.coph.2003.08.001

Cande, J., Prud'homme, B., and Gompel, N. (2013). Smells like evolution: the role of chemoreceptor evolution in behavioral change. Curr. Opin. Neurobiol. 23, 152-158. doi: 10.1016/j.conb.2012.07.008

Carey, A. F., Wang, G., Su, C.-Y., Zwiebel, L. J., and Carlson, J. R. (2010). Odorant reception in the malaria mosquito Anopheles gambiae. Nature 464, 66-71. doi: $10.1038 /$ nature 08834

Chavez-Noriega, L. E., Crona, J. H., Washburn, M. S., Urrutia, A., Elliott, K. J., and Johnson, E. C. (1997). Pharmacological characterization of recombinant human neuronal nicotinic acetylcholine receptors h alpha 2 beta 2 , h alpha 2 beta 4 , h alpha 3 beta 2 , h alpha 3 beta 4 , h alpha 4 beta 2 , h alpha 4 beta 4 and $\mathrm{h}$ alpha 7 expressed in Xenopus oocytes. J. Pharmacol. Exp. Ther. 280, 346-356.

Clements, J. D. (1996). Transmitter timecourse in the synaptic cleft: its role in central synaptic function. Trends Neurosci. 19, 163-171. doi: 10.1016/S01662236(96)10024-2

Croset, V., Rytz, R., Cummins, S. F., Budd, A., Brawand, D., Kaessmann, H., et al. (2010). Ancient protostome origin of chemosensory ionotropic glutamate receptors and the evolution of insect taste and olfaction. PLoS Genet. 6:e1001064. doi: 10.1371/journal.pgen.1001064

de Lacy Costello, B., Amann, A., Al-Kateb, H., Flynn, C., Filipiak, W., Khalid, T., et al. (2014). A review of the volatiles from the healthy human body. J. Breath Res. 8:014001. doi: 10.1088/1752-7155/8/1/014001

Dickens, J. C. (1990). Specialized receptor neurons for pheromones and host plant odors in the boll weevil, Anthonomus grandis Boh. (Coleoptera: Curculionidae). Chem. Senses. 15, 311-331. doi: 10.1093/chemse/15.3.311

Dobritsa, A., van der Goes van Naters, W., Warr, C., Steinbrecht, R., and Carlson, J. (2003). Integrating the molecular and cellular basis of odor coding in the Drosophila antenna. Neuron 37, 827-841. doi: 10.1016/S0896-6273(03) 00094-1

Dudareva, N., Negre, F., Nagegowda, D. A., and Orlova, I. (2006). Plant volatiles: recent advances and future perspectives. Crit. Rev. Plant Sci. 25, 417-440. doi: $10.1080 / 07352680600899973$

Faisal, A. A., Selen, L. P. J., and Wolpert, D. M. (2008). Noise in the nervous system. Nat. Rev. Neurosci. 9, 292-303. doi: 10.1038/nrn2258
Felten, S. Y., and Olschowka, J. (1987). Noradrenergic sympathetic innervation of the spleen: II. Tyrosine hydroxylase (TH)-positive nerve terminals form synapticlike contacts on lymphocytes in the splenic white pulp. J. Neurosci. Res. 18, 37-48. doi: 10.1002/jnr.490180108

Firestein, S. (2001). How the olfactory system makes sense of scents. Nature 413, 211-218. doi: 10.1038/35093026

Gardiner, A., Barker, D., and Butlin, R. (2008). Drosophila chemoreceptor gene evolution: selection, specialization and genome size. Mol. Ecol. 17, 1648-1657. doi: 10.1111/j.1365-294X.2008.03713.x

Große-Wilde, E., Gohl, T., Bouché, E., Breer, H., and Krieger, J. (2007). Candidate pheromone receptors provide the basis for the response of distinct antennal neurons to pheromonal compounds. Eur. J. Neurosci. 25, 2364-2373. doi: 10.1111/j.1460-9568.2007.05512.x

Hallem, E. A., and Carlson, J. R. (2004). The odor coding system of Drosophila. Trends Genet. 20, 453-459. doi: 10.1016/j.tig.2004.06.015

Hallem, E. A., and Carlson, J. R. (2006). Coding of odors by a receptor repertoire. Cell 125, 143-160. doi: 10.1016/j.cell.2006.01.050

Hallem, E. A., Ho, M. G., and Carlson, J. R. (2004). The molecular basis of odor coding in the Drosophila antenna. Cell 117, 965-979. doi: 10.1016/j.cell.2004.05.012

Hansson, B. S., and Stensmyr, M. C. (2011). Evolution of insect olfaction. Neuron 72, 698-711. doi: 10.1016/j.neuron.2011.11.003

Harper, R. J., Almirall, J. R., and Furton, K. G. (2005). Identification of dominant odor chemicals emanating from explosives for use in developing optimal training aid combinations and mimics for canine detection. Talanta 67, 313-327. doi: 10.1016/j.talanta.2005.05.019

Hill, C. A., Fox, A. N., Pitts, R. J., Kent, L. B., Tan, P. L., Chrystal, M. A., et al. (2002). G protein-coupled receptors in Anopheles gambiae. Science 298, 176-178. doi: 10.1126/science.1076196

Hughes, D. T., Pelletier, J., Luetje, C. W., and Leal, W. S. (2010). Odorant receptor from the southern house mosquito narrowly tuned to the oviposition attractant skatole. J. Chem. Ecol. 36, 797-800. doi: 10.1007/s10886-010-9828-9

Kafka, W. A. (1987). Similarity of reaction spectra and odor discrimination - Single receptor cell recordings in Antheraea polyphemus (Saturniidae). J. Comp. Physiol. A 161, 867-880. doi: 10.1007/BF00610228

Karayannis, T., Elfant, D., Huerta-Ocampo, I., Teki, S., Scott, R. S., Rusakov, D. A., et al. (2010). Slow GABA transient and receptor desensitization shape synaptic responses evoked by hippocampal neurogliaform cells. J. Neurosci. 30, 9898-9909. doi: 10.1523/JNEUROSCI.5883-09.2010

Knudsen, J. T., Eriksson, R., Gershenzon, J., and Ståhl, B. (2006). Diversity and distribution of floral scent. Bot. Rev. 72, 1-120. doi: 10.1663/00068101(2006)72[1:DADOFS]2.0.CO;2

Kreher, S. A., Mathew, D., Kim, J., and Carlson, J. R. (2008). Translation of sensory input into behavioral output via an olfactory system. Neuron 59, 110-124. doi: 10.1016/j.neuron.2008.06.010

Kuffler, S. W., and Yoshikami, D. (1975). The number of transmitter molecules in a quantum: an estimate from iontophoretic application of acetylcholine at the neuromuscular synapse. J. Physiol. 251, 465-482. doi: 10.1113/jphysiol.1975.sp011103

Kvist, T., Hansen, K. B., and Bräuner-Osborne, H. (2011). The use of Xenopus oocytes in drug screening. Expert Opin. Drug Discov. 6, 141-153. doi: 10.1517/17460441.2011.546396

Leary, G. P., Allen, J. E., Bunger, P. L., Luginbill, J. B., Linn, C. E., Macallister, I. E., et al. (2012). Single mutation to a sex pheromone receptor provides adaptive specificity between closely related moth species. Proc. Natl. Acad. Sci. U.S.A. 109, 14081-14086. doi: 10.1073/pnas.1204661109

Leon, M., and Johnson, B. A. (2003). Olfactory coding in the mammalian olfactory bulb. Brain Res. Rev. 42, 23-32. doi: 10.1016/S0165-0173(03) 00142-5

Liu, C., Liu, Y., Guo, M., Cao, D., Dong, S., and Wang, G. (2014). Narrow tuning of an odorant receptor to plant volatiles in Spodoptera exigua (Hübner). Insect Mol. Biol. 23, 487-496. doi: 10.1111/imb.12096

Liu, Y., Liu, C., Lin, K., and Wang, G. (2013). Functional specificity of sex pheromone receptors in the cotton bollworm Helicoverpa armigera. PLoS ONE 8:e62094. doi: 10.1371/journal.pone.0062094

Malnic, B., Hirono, J., Sato, T., and Buck, L. B. (1999). Combinatorial receptor codes for odors. Cell 96, 713-723. doi: 10.1016/S0092-8674(00) 80581-4 
Mandairon, N., and Linster, C. (2008). Odor perception and olfactory bulb plasticity in adult Mammals. J. Neurophysiol. 101, 2204-2209. doi: 10.1152/jn.00076.2009

McBride, C. (2007). Rapid evolution of smell and taste receptor genes during host specialization in Drosophila sechellia. Proc. Natl. Acad. Sci. U.S.A. 104, 4996-5001. doi: 10.1073/pnas.0608424104

McBride, C. S., Arguello, J. R., and O'Meara, B. C. (2007). Five Drosophila genomes reveal nonneutral evolution and the signature of host specialization in the chemoreceptor superfamily. Genetics 177, 1395-1416. doi: 10.1534/genetics.107.078683

McBride, C. S., Baier, F., Omondi, A. B., Spitzer, S. A., Lutomiah, J., Sang, R., et al. (2014). Evolution of mosquito preference for humans linked to an odorant receptor. Nature 515, 222-227. doi: 10.1038/nature13964

Missbach, C., Dweck, H. K., Vogel, H., Vilcinskas, A., Stensmyr, M. C., Hansson, B. S., et al. (2014). Evolution of insect olfactory receptors. Elife 3:e02115. doi: 10.7554/eLife.02115

Mitsuno, H., Sakurai, T., Murai, M., Yasuda, T., Kugimiya, S., Ozawa, R., et al. (2008). Identification of receptors of main sex-pheromone components of three Lepidopteran species. Eur. J. Neurosci. 28, 893-902. doi: 10.1111/j.14609568.2008.06429.x

Miura, N., Nakagawa, T., Tatsuki, S., Touhara, K., and Ishikawa, Y. (2009). A malespecific odorant receptor conserved through the evolution of sex pheromones in Ostrinia moth species. Int. J. Biol. Sci. 5, 319-330. doi: 10.7150/ijbs.5.319

Moncrieff, R. W. (1949). What is odor? A new theory. Am. Perfumer. 54, 453-454.

Nakagawa, T., Sakurai, T., Nishioka, T., and Touhara, K. (2005). Insect sexpheromone signals mediated by specific combinations of olfactory receptors. Science 307, 1638-1642. doi: 10.1126/science.1106267

Nakanishi, N., Axel, R., and Shneider, N. A. (1992). Alternative splicing generates functionally distinct N-methyl-D-aspartate receptors. Proc. Natl. Acad. Sci. U.S.A. 89, 8552-8556. doi: 10.1073/pnas.89.18.8552

Neafsey, D. E., Waterhouse, R. M., Abai, M. R., Aganezov, S. S., Alekseyev, M. A., Allen, J. E., et al. (2015). Highly evolvable malaria vectors: the genomes of 16 Anopheles mosquitoes. Science 347, 1258522-1258522. doi: 10.1126/science. 1258522

Nei, M., Niimura, Y., and Nozawa, M. (2008). The evolution of animal chemosensory receptor gene repertoires: roles of chance and necessity. Nat. Rev. Genet. 9, 951-963. doi: 10.1038/nrg2480

Pelletier, J., Hughes, D. T., Luetje, C. W., and Leal, W. S. (2010). An odorant receptor from the southern house mosquito Culex pipiens quinquefasciatus sensitive to oviposition attractants. PLoS ONE 5:e10090. doi: 10.1371/journal.pone. 0010090

Penn, D. J., Oberzaucher, E., Grammer, K., Fischer, G., Soini, H. A., Wiesler, D., et al. (2007). Individual and gender fingerprints in human body odour. J. $R$. Soc. Interface 4, 331-340. doi: 10.1098/rsif.2006.0182

Phillips, M. (1997). Method for the collection and assay of volatile organic compounds in breath. Anal. Biochem. 247, 272-278. doi: 10.1006/abio. 1997.2069

Phillips, M., Cataneo, R. N., Chaturvedi, A., Kaplan, P. D., Libardoni, M., Mundada, M., et al. (2013). Detection of an extended human volatome with comprehensive two-dimensional gas chromatography time-of-flight mass spectrometry. PLoS ONE 8:e75274. doi: 10.1371/journal.pone.0075274

Ray, A., van Naters, W. G., and Carlson, J. R. (2014). Molecular determinants of odorant receptor function in insects. J. Biosci. 39, 555-563. doi: 10.1007/s12038014-9447-7

Rinaldi, A. (2007). The scent of life. EMBO Rep. 8, 629-633. doi: 10.1038/sj.embor.7401029

Rinker, D. C., Jones, P. L., Pitts, R. J., Rutzler, M., Camp, G., Sun, L., et al. (2012). Novel high-throughput screens of Anopheles gambiae odorant receptors reveal candidate behaviour-modifying chemicals for mosquitoes. Physiol. Entomol. 37, 33-41. doi: 10.1111/j.1365-3032.2011.00821.x

Robertson, H. M., and Wanner, K. W. (2006). The chemoreceptor superfamily in the honey bee, Apis mellifera: expansion of the odorant, but not gustatory, receptor family. Genome Res. 16, 1395-1403. doi: 10.1101/gr.5057506

Sachse, S., and Galizia, C. G. (2003). The coding of odour-intensity in the honeybee antennal lobe: local computation optimizes odour representation. Eur. J. Neurosci. 18, 2119-2132. doi: 10.1046/j.1460-9568.2003.02931.x

Sánchez-Gracia, A., Vieira, F. G., and Rozas, J. (2009). Molecular evolution of the major chemosensory gene families in insects. Heredity 103, 208-216. doi: 10.1038/hdy.2009.55
Sato, K., Tanaka, K., and Touhara, K. (2011). Sugar-regulated cation channel formed by an insect gustatory receptor. Proc. Natl. Acad. Sci. U.S.A. 108, 11680-11685. doi: 10.1073/pnas.1019622108

Schneider, D. (1964). Insect antennae. Ann. Rev. Entomol. 9, 103-122. doi: 10.1146/annurev.en.09.010164.000535

Silbering, A. F., Okada, R., Ito, K., and Galizia, C. G. (2008). Olfactory information processing in the Drosophila antennal lobe: anything goes? J. Neurosci. 28, 13075-13087. doi: 10.1523/JNEUROSCI.2973-08.2008

Smit, A. B., Syed, N. I., Schaap, D., van Minnen, J., Klumperman, J., Kits, K. S., et al. (2001). A glia-derived acetylcholine-binding protein that modulates synaptic transmission. Nature 411, 261-268. doi: 10.1038/35077000

Stensmyr, M. C., Dweck, H., Farhan, A., Ibba, I., and Strutz, A. (2012). A Conserved dedicated olfactory circuit for detecting harmful microbes in Drosophila. Cell 151, 1345-1357. doi: 10.1016/j.cell.2012.09.046

Stocker, R., and Nuesch, H. (1975). Ultrastructural studies on neuromuscular contacts and the formation of junctions in the flight muscle of Antheraea polyphemus (Lep.) I. Normal adult development. Cell Tissue Res. 159, 245-266. doi: $10.1007 /$ BF00219160

Suer, R. A. (2014). Unravelling the Malaria Mosquito's Sense of Smell: Neural and Behavioural Responses to Human-Derived Compounds, 2011, Ph.D. Thesis, Wageningen University.

Suh, E., Bohbot, J., and Zwiebel, L. J. (2014). Peripheral olfactory signaling in insects. Curr. Opin. Insect Sci. 6, 86-92. doi: 10.1016/j.cois.2014.10.006

Sun, M., Liu, Y., Walker, W. B., Liu, C., Lin, K., Gu, S., et al. (2013). Identification and characterization of pheromone receptors and interplay between receptors and pheromone binding proteins in the diamondback moth, Plutella xyllostella. PLoS ONE 8:e62098. doi: 10.1371/journal.pone.0062098

Tanaka, K., Uda, Y., Ono, Y., Nakagawa, T., Suwa, M., Yamaoka, R., et al. (2009). Highly selective tuning of a silkworm olfactory receptor to a key mulberry leaf volatile. Curr. Biol. 19, 881-890. doi: 10.1016/j.cub.2009.04.035

Vogt, R., and Riddiford, L. (1981). Pheremone binding and inactivation by moth antennae. Nature 293, 161-163. doi: 10.1038/293161a0

Wang, G., Carey, A. F., Carlson, J. R., and Zwiebel, L. J. (2010). Molecular basis of odor coding in the malaria vector mosquito Anopheles gambiae. Proc. Natl. Acad. Sci. U.S.A. 107, 4418-4423. doi: 10.1073/pnas.0913392107

Wang, G., Vásquez, G. M., Schal, C., Zwiebel, L. J., and Gould, F. (2011). Functional characterization of pheromone receptors in the tobacco budworm Heliothis virescens. Insect Mol. Biol. 20, 125-133. doi: 10.1111/j.1365-2583.2010.01045.x

Wanner, K. W., Nichols, A. S., Allen, J. E., Bunger, P. L., Garczynski, S. F., Linn, C. E., et al. (2010). Sex pheromone receptor specificity in the European corn borer moth, Ostrinia nubilalis. PLoS ONE 5:e8685. doi: 10.1371/journal.pone. 0008685

Wanner, K. W., Nichols, A. S., Walden, K. K. O., Brockmann, A., Luetje, C. W., and Robertson, H. M. (2007). A honey bee odorant receptor for the queen substance 9-oxo-2-decenoic acid. Proc. Natl. Acad. Sci. U.S.A. 104, 14383-14388. doi: 10.1073/pnas.0705459104

Wilson, D. A., and Stevenson, R. J. (2003). Olfactory perceptual learning: the critical role of memory in odor discrimination. Neurosci. Biobehav. Rev. 27, 307-328. doi: 10.1016/S0149-7634(03)00050-2

Wonnacott, S., and Barik, J. (2007). Nicotinic ACh receptors. Tocris Biosci. Sci. Rev. $28,1-20$.

Xu, P., Garczynski, S. F., Atungulu, E., Syed, Z., Choo, Y.-M., Vidal, D. M., et al. (2012). Moth sex pheromone receptors and deceitful parapheromones. PLoS ONE 7:e41653. doi: 10.1371/journal.pone.0041653

Zhou, X., Slone, J. D., Rokas, A., Berger, S. L., Liebig, J., Ray, A., et al. (2012). Phylogenetic and transcriptomic analysis of chemosensory receptors in a pair of divergent ant species reveals sex-specific signatures of odor coding. PLoS Genet. 8:e1002930. doi: 10.1371/journal.pgen.1002930

Conflict of Interest Statement: The authors declare that the research was conducted in the absence of any commercial or financial relationships that could be construed as a potential conflict of interest.

Copyright (C) 2015 Bohbot and Pitts. This is an open-access article distributed under the terms of the Creative Commons Attribution License (CC BY). The use, distribution or reproduction in other forums is permitted, provided the original author(s) or licensor are credited and that the original publication in this journal is cited, in accordance with accepted academic practice. No use, distribution or reproduction is permitted which does not comply with these terms. 